\title{
Modeling Laser-Induced Incandescence of Soot Particles Produced In A Premixed $\mathrm{Ch}_{4} / \mathrm{O}_{2} / \mathrm{N}_{2}$ Flat Flame
}

\author{
Sébastien Menanteau ${ }^{1}$, Romain Lemaire ${ }^{2}$ \\ ${ }^{1}$ Energy, Environment \& Materials Engineering, ICAM Lille, 59016, France \\ sebastien.menanteau@icam.fr \\ ${ }^{2}$ TFT laboratory, Department of Mechanical Engineering, École de technologie supérieure \\ Montréal, Québec, H3C 1K3, Canada \\ romain.lemaire@etsmtl.ca
}

\begin{abstract}
The present work deals with the modeling of an extensive set of laser-induced incandescence (LII) signals collected in an atmospheric $\mathrm{CH}_{4} / \mathrm{O}_{2} / \mathrm{N}_{2}$ premixed flat flame. To do so, a refined LII model built upon a comprehensive version of soot heat- and massbalance equations has been used. This latter especially includes an absorption sub-model accounting for saturation of linear, single- and multi-photon absorption processes together with terms standing for soot oxidation and particle cooling by sublimation, conduction, radiation and thermionic emission. The comparison between the simulated and the experimentally-monitored data reported herein has been achieved based on LII fluence curves and time-decays obtained at different heights above the burner as well as on the basis of measured soot temperature profiles as a function of the laser fluence. In terms of results, the theoretical model implemented within this study turns out to correctly reproduce measured LII fluence curves and soot temperature profiles although it fails to correctly simulate LII time decays collected at high fluences. An analysis of the possible improvements to be operated to the so-implemented model eventually led to the conclusion that integrating an additional energy flux standing for soot annealing could allow obtaining improved agreements between theoretical and measured signals thus giving interesting insights for complementary works to be undertaken.
\end{abstract}

Keywords: soot, laser-induced incandescence, modeling, absorption function, annealing

\section{Introduction}

Soot emissions are currently pointed out as being responsible for major environmental and health issues. Numerous works have therefore been undertaken during more than a half century to elucidate the main mechanisms underlying the soot formation, growth and oxidation processes [1]. Understanding and predicting these fundamental phenomena, however, requires using and/or developing advanced in situ diagnostics allowing complex reacting media to be probed [1,2]. Within this context, laser-induced incandescence (LII) has become a workhorse for the detection of combustion-generated nanoparticles in wide varied environments including lab-scale flames and exhaust gases from engines or turbines $[\mathbf{3 , 4}]$. This technique briefly consists in heating soot by means of a high-power pulsed-laser source up to temperatures high enough to allow the emission of a measureable quasi-blackbody radiation. This latter can then be collected using a photomultiplier tube (for time-resolved analyses) or an intensified ICCD camera (for spatially resolved measurements). Since Melton showed that the magnitude of LII signals could be considered as proportional to the particle volume fraction in the probed medium for detection wavelengths comprised in the visible range [5], LII has been extensively used for soot concentration measurements [6] while being also demonstrated as being a very powerful diagnostic for particle size [7] and optical properties [8] assessment. That being said, correctly measuring and interpreting soot incandescence signals still require a firm understanding of the physical processes governing the LII phenomenon thus explaining why considerable efforts have been devoted over the past decades to the development of predictive models capable of simulating the radiative emission from laser-heated particles under a wide range of operating conditions [4,9]. Among important contributions to the field, one can cite the work from Weeks and Duley who proposed the first energy-balance model aiming at simulating the time-dependent light emission from aerosols of submicron powders of carbon black and alumina excited by a pulsed $\mathrm{CO}_{2}$ laser [10]. Eckbreth [11] and Burakov et al. [12] then proposed improved models integrating equations standing for sublimation of heated soot which subsequently led Melton to propose a model formulation based on the coupling of energy- and mass-balance equations incorporating terms standing for absorption of the laser energy and soot cooling by conduction, radiation and sublimation 
[5]. Since then, almost all the LII models proposed in the literature have been built upon a similar pattern. They actually predict the radiative emission from particles by introducing the temporal evolutions of soot temperature and diameter (these latter being issued from the solving of the coupled differential equations standing for particle energy and mass balances) into a Planck function. The main differences between existing models actually lies in the nature of the energy fluxes considered within the balance equations and in the formulation of the governing equations accounting for such fluxes. The reason for that can be traced to the developments that have been achieved over the past years in the formulation of improved sublimation $[\mathbf{1 3 , 1 4}]$ and conduction sub-models $[\mathbf{1 5 , 1 6}]$ noting that the most important contributions to LII model refinement are probably those brought by Michelsen who proposed implementing mechanisms accounting for soot melting, annealing and oxidation, thermionic emissions, non-thermal photodesorption of carbon clusters from the particle surface and multi-photon absorption leading to the photodesorption of $\mathrm{C}_{2}$ clusters at high fluences $[\mathbf{9 , 1 4}$. The use of such a type of refined model has been particularly shown in [17] to allow predicting signals merging on a single curve with those measured in co-flow diffusion flames of ethylene. In a subsequent modeling work based on the use of inverse techniques, Lemaire and Mobtil [18] demonstrated that including photolytic mechanisms such as those proposed by Michelsen was quite required to be able to simulate LII signals over a wide range of fluences. While strengthening the consistency of the modeling approach developed in [9,14], this work, however, led to the conclusion that an in-depth analysis of the formulation and parameterization of the sub-models embedded within Michelsen-derived simulation tools was still necessary as the equations and parameters reported in $[\mathbf{9 , 1 4}]$ turned out to be unsuitable to reproduce the experimental data obtained by Goulay et al. in a diffusion flame of ethylene [19]. Lemaire and Mobtil eventually highlighted the need for additional theoretical analyses supported by comparisons between simulated data and experimentally monitored signals obtained in well characterized media. With this in mind and considering that the validity of the database provided by Goulay et al. has been recently somewhat questioned by Mansmann et al. [20], Menanteau and Lemaire proposed a series of works [21,22] in which a set of LII signals measured in a diffusion flame of Diesel has been simulated using one of the most (if not the most) refined LII-model formulation ever implemented so far. By using an original optimization procedure coupling design of experiments and a genetic algorithm-based solver, these authors proposed a fully parameterized LII model formulation allowing obtaining simulated and measured signals merging on a single curve. They moreover drew conclusions regarding the consistency of currently used conduction sub-models [21] while deriving values of the thermal accommodation coefficient and the particle-maturity-dependent soot absorption function [22]. As a continuation of this research, it is proposed in the present work to model a comprehensive database of LII signals measured in a laminar-rich-premixed flat flame of $\mathrm{CH}_{4} / \mathrm{O}_{2} / \mathrm{N}_{2}$ [23]. In addition to providing thoroughly acquired data in a well characterized combustion media, the interest of selecting the results from [23] also lies in the fact that Bejaoui et al. also tried to model the signals they collected using a refined LII model derived from the formulations proposed by Michelsen. They, however, did not obtain simulated data showing a satisfying agreement with measured ones thus prompting the need for additional modeling works to be conducted as proposed herein by taking advantage of the recent developments carried out in $[\mathbf{2 1 , 2 2}$. After briefly presenting the database as well as the modeling approach used within this study, a comparison between predicted and measured LII responses will be achieved. A specific attention will finally be devoted to the improvements that could be brought to the LII-modeling tool implemented in this work so as to increase its predictive capability.

\section{Methodology}

\subsection{Experimental Database}

The experimental results used for the purposes of the present study are issued from the extensive data set proposed by Bejaoui et al. in [23]. This comprehensive work that couples experimental and numerical analyses gathers a series of LII time decays collected at different heights above the burner (HAB) in an atmospheric $\mathrm{CH}_{4} / \mathrm{O}_{2} / \mathrm{N}_{2}$ premixed flat flame. As detailed in [23], such signals have been obtained using a 1064-nm nearly top-hat laser beam and a detection wavelength of $610 \mathrm{~nm}$. Averaged temperatures of soot over a 10-ns period after the peak of the laser pulse have moreover been reported therein based on the analysis of spectrally-resolved LII radiations collected on a 500- to 630-nm wavelength range. In addition to time-resolved LII signals and average soot temperature profiles collected at different 
$\mathrm{HAB}$ and for wide varied laser fluences, this detailed database also includes spatial- and temporal-profiles of the laser energy, NO-LIF thermometry-derived flame temperatures as well as information regarding primary particle and aggregate sizes as determined by transmission electron microscopy. This is therefore such a comprehensive set of thoroughly acquired data that has been selected to be modeled within the framework of the present theoretical analysis.

\subsection{Modeling Approach}

Following the modeling approach previously adopted in $[\mathbf{2 1 , 2 2}]$, the selected LII simulation tool has been built considering mechanisms accounting for particle heating by absorption of the laser energy $\left(\dot{Q}_{a b s}\right)$, soot annealing $\left(\dot{Q}_{\text {ann }}\right)$ and oxidation $\left(\dot{Q}_{o x}\right)$ together with cooling processes by radiation $\left(\dot{Q}_{\text {rad }}\right)$, thermionic emission $\left(\dot{Q}_{t h}\right)$ sublimation $\left(\dot{Q}_{s u b}\right)$ and conduction $\left(\dot{Q}_{\text {cond }}\right)$. Based on such mechanisms that are likely to modify the particle energy and mass, one can build a system

of coupled differential equations depicting the variations of the soot internal energy rate $\left(\frac{d U_{i n t}}{d t}\right)$ and mass $\left(\frac{d M_{p}}{d t}\right)$ as a function of time as depicted by equations (1) and (2):

$$
\begin{gathered}
\frac{d U_{i n t}}{d t}=\dot{Q}_{a b s}+\dot{Q}_{a n n}+\dot{Q}_{o x}-\dot{Q}_{r a d}-\dot{Q}_{t h}-\dot{Q}_{s u b}-\dot{Q}_{c o n d} \\
\frac{d M_{p}}{d t}=\sum_{j=1}^{5}\left(\frac{d M_{p}}{d t}\right)_{s u b, j}+\left(\frac{d M_{p}}{d t}\right)_{o x}
\end{gathered}
$$

where subscripts 'sub' and 'ox' denote the contributions of the sublimation and oxidation mechanisms to the mass loss, respectively, while ' $\mathrm{j}$ ' stands for the contribution of each vaporized carbon cluster $\mathrm{C}_{\mathrm{j}}$ to the particle mass loss. As far as $\frac{d U_{i n t}}{d t}$, $\dot{Q}_{a n n}$ and $\dot{Q}_{r a d}$ are concerned, they have been implemented as reported in [14] while $\dot{Q}_{t h}$ and $\dot{Q}_{o x}$ have been formulated, for their part, as detailed in [9] and [24], respectively. Concerning the modeling of the conduction flux $\dot{Q}_{\text {cond }}$, a Fuchs approach [25] that covers the entire range of heat conduction regimes has been especially selected as recommended in [15]. It briefly consists in determining the temperature $T_{\delta}$ and the characteristic length $\delta$ of a delimiting sphere separating the free molecular $(F M)$ regime from the continuum $(C)$ one, both conduction modes being characterized by energy dissipation rates that can be expressed as follows:

$$
\begin{gathered}
\dot{Q}_{\text {cond }, F M}=\frac{1}{8} \cdot \pi \cdot \alpha_{T} \cdot D_{H C}{ }^{2} \cdot P_{g} \cdot \sqrt{\frac{8 \cdot k_{B} \cdot T_{\delta}}{\pi \cdot M_{g}}} \cdot \frac{\gamma^{*}+1}{\gamma^{*}-1} \cdot\left(\frac{T_{p}}{T_{\delta}}-1\right) \\
\dot{Q}_{c o n d, C}=4 \cdot \pi \cdot\left(\frac{D_{H C}}{2}+\delta\right) \cdot \int_{T_{g}}^{T_{\delta}} k_{g}(T) d T
\end{gathered}
$$

where $\alpha_{T}$ represents the thermal accommodation coefficient (the value of which has been set as detailed in section 3 ), $D_{H C}$ stands for the equivalent heat conduction diameter defined in [16], $P_{g}$ is the ambient gas pressure, $k_{B}$ denotes the Boltzmann constant $\left(1.381 \cdot 10^{-23} \mathrm{~J} \cdot \mathrm{K}^{-1}\right), M_{g}$ is the average mass of gas molecules, $\gamma^{*}$ is the mean heat capacity ratio as expressed in [16], $T_{p}$ represents the soot particle temperature while $T_{g}$ and $k_{g}$ are the temperature and the heat conduction coefficient of the surrounding gas, respectively. As far as the absorption flux is concerned, it has been expressed following equation (5) that allows accounting for saturation of linear, single- and multi-photon absorption processes of both unannealed and annealed soot fractions:

$$
\dot{Q}_{a b s, r}=C_{a b s, r} \cdot \frac{f_{1, r} \cdot B_{\lambda 1, r}}{\int_{0}^{t_{l}} q_{\exp }(t) d t} \cdot\left\{1-\exp \left[-\frac{F \cdot q_{\exp }(t)}{B_{\lambda 1, r}}\right]\right\}+\frac{n \cdot h \cdot c}{\lambda_{l}} \cdot k_{\lambda n, r}
$$

where the subscript ' $r$ ' stands for either unannealed and annealed parts of soot (denoted with subscripts ' $s$ ' and 'a', respectively, in the following), $f_{1, r}$ and $B_{\lambda 1, r}$ correspond to empirical factors related to the single-photon absorption process, $q_{\text {exp }}, t_{l}, \lambda_{l}$ and $F$ are the normalized irradiance, pulse duration, energy density and excitation wavelength related to the laser, 
respectively, $h$ and $c$ stand for the Planck constant $\left(6.62 \cdot 10^{-34} \mathrm{~J} \cdot \mathrm{s}\right)$ and the speed of light $\left(2.998 \cdot 10^{10} \mathrm{~cm} \cdot \mathrm{s}^{-1}\right)$ while $n$ represents the number of photons to be adsorbed to photodesorb $\mathrm{C}_{2}$ clusters. Concerning the soot absorption cross-section $C_{a b s, r}$, it can be put into equation in the form:

$$
C_{a b s, s}=\left(1-X_{a}\right) \cdot \frac{\pi^{2} \cdot D_{p}{ }^{3}}{\lambda_{l}} \cdot E(m) \& C_{a b s, a}=X_{a} \cdot \frac{\pi^{2} \cdot D_{p}{ }^{3}}{\lambda_{l}} \cdot f_{a} \cdot E_{a}(m)
$$

where $X_{a}$ represents the soot annealed fraction, $D_{p}$ is the primary particle diameter and $f_{a}$ corresponds to an empirical scaling factor for annealed soot [14]. While the absorption function of annealed soot $E_{a}(m)$ has been expressed as proposed in [14], its counterpart for unannealed particles $E(m)$ has been set as a function of the soot maturity stage through the fitting procedure described in section 3. Concerning the rate constant for removal of $\mathrm{C}_{2}$ clusters by photodesorption $\left(k_{\lambda n, r}\right)$ which is integrated within $\dot{Q}_{a b s}$ and $\dot{Q}_{s u b}$ expressions, it has been formulated following [22]:

$$
k_{\lambda n, r}=X_{s / a} \cdot \frac{\lambda_{l}}{n \cdot h \cdot c} \cdot \frac{\sigma_{\lambda n, r} \cdot \pi \cdot D_{p}{ }^{3} \cdot N_{s r}}{6} \cdot \frac{\left(B_{\lambda n, r}\right)^{n}}{\int_{0}^{t_{\infty}}\left[q_{\exp }(t)\right]^{n} d t} \cdot\left\{1-\exp \left[-\left(\frac{F \cdot q_{\exp }(t)}{B_{\lambda n, r}}\right)^{n}\right]\right\}
$$

where $X_{s / a}$ is equal to either $1-X_{a}$ or $X_{a}$ for unannealed and annealed soot fractions, respectively, $\sigma_{\lambda n, r}$ represents the multiphoton absorption cross-section for the photodesorption of $\mathrm{C}_{2}$ clusters, $N_{s r}$ is the density of carbon atoms on the surface of primary particles $\left(N_{s S}=2.8 \cdot 10^{15} \mathrm{~cm}^{-2}\right.$ and $N_{s a}=3.8 \cdot 10^{15} \mathrm{~cm}^{-2}$ [14]) while $B_{\lambda n, r}$ is an empirical saturation coefficient for multiphoton absorption. The solving of the system of differential equations depicting the time-dependence of the soot internal energy rate and mass allowed deriving the variations of $T_{p}$ and $D_{p}$ as a function of time, the values of which have then been introduced into a Planck function integrated over the spectral range of the detection system (including its spectral response as detailed in $[\mathbf{1 8 , 2 1 , 2 2 ]})$ to obtain theoretical LII signals. One can finally add that the results of the experimental characterization carried out in [23] have been used to correctly set the model input parameters such as the temperature of the surrounding gas and the size distributions of the primary particles at the different investigated HAB. Internal multi-scattering likewise shielding effect that may occur within aggregates have eventually been neglected herein considering the small number of primary particles per aggregate experimentally monitored in [23].

\section{Results and Discussion}

The comparison between measured and simulated results has been achieved based on LII time decays and fluence curves as well as on the basis of soot temperature profiles plotted as a function of the laser fluence. For the sake of completeness, the modeling results from [23] (i.e. time-resolved LII signals and average temperatures of soot over 10 ns after the peak of the laser pulse) have also been reported hereafter to figure out whether the model proposed in the present work allows better simulating experimentally monitored data or not. To give a brief description of the calculation procedure adopted in [23], one may mention that two models have in fact been implemented. The first one (referenced as "base-model") integrates expressions commonly used to account for laser absorption, soot internal energy change, heat conduction and sublimation. This basic simulation tool (whose detailed description is given in [9,16]) did not allow satisfactorily reproducing measured time-resolved LII signals and averaged soot temperatures, however. Bejaoui et al. thus considered an extended model formulation derived from the work of Michelsen $[\mathbf{9 , 1 4}$ especially as far as the expression of the sublimation flux is concerned. Annealing, oxidation and thermionic emission have not been considered, however, as is the case of the photolytic mechanisms involved in the absorption flux (the importance of which has still been demonstrated in [18]) so as to isolate the impact of the sublimation sub-model on the LII responses predicted by the base- and the extended-models. As this second formulation allowed improving the agreement between modeled- and measured-LII time decays, it has thus been considered for the comparison analysis proposed below. Besides and concerning the refined model implemented for the purposes of the present study, this latter has been parameterized as detailed in $[\mathbf{2 1 , 2 2}]$. Only the soot-maturity-dependent absorption function, the multiphoton absorption cross-section for $\mathrm{C}_{2}$ cluster photodesorption and the thermal accommodation coefficient have been adjusted as compared to $[\mathbf{2 1 , 2 2}]$ so as to take into account the specific nature of the investigated combustion medium (a premixed $\mathrm{CH}_{4} / \mathrm{O}_{2} / \mathrm{N}_{2}$ flat flame that significantly diverges from the Diesel flame probed in $[\mathbf{2 1 , 2 2 ]})$. Eventually and to ease the comparison 
with the simulated data from [23], the annealing sub-model has been deactivated in a first stage, noting that the impact of such an energy flux will be discussed more in detail at the end of this section. In terms of results, one can first note that a perfect fit between measured and simulated fluence curves can be obtained when fixing $E(m)$ values ranging from 0.2 to 0.42 for HAB comprised between 7 and $15 \mathrm{~mm}$ as shown in Fig. 1.

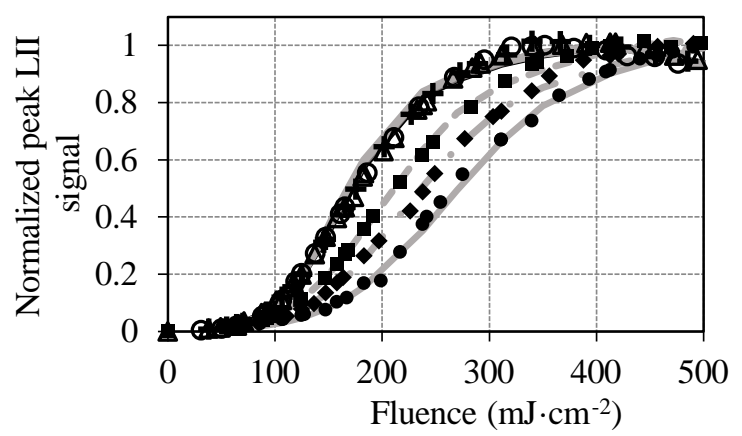

- $\quad$ exp. [23], 7 mm HAB

- exp. [23], $8 \mathrm{~mm}$ HAB

- $\quad$ exp. [23], $9 \mathrm{~mm}$ HAB

$\Delta \quad$ exp. [23], $13 \mathrm{~mm}$ HAB

$+\quad$ exp. [23], 14 mm HAB

o exp. [23], $15 \mathrm{~mm} \mathrm{HAB}$

model, $7 \mathrm{~mm} \mathrm{HAB}, \mathrm{E}(\mathrm{m})=0.2$

- model, $8 \mathrm{~mm}$ HAB, $\mathrm{E}(\mathrm{m})=0.25$

$-\infty$ model, $9 \mathrm{~mm}$ HAB, $\mathrm{E}(\mathrm{m})=0.3$

model, $13 \mathrm{~mm} \mathrm{HAB}, \mathrm{E}(\mathrm{m})=0.39$

model, 14 mm HAB, $\mathrm{E}(\mathrm{m})=0.41$

model, $15 \mathrm{~mm}$ HAB, $\mathrm{E}(\mathrm{m})=0.42$

Fig. 1: Comparison between measured and simulated LII fluence curves obtained at different HAB.

Such an increase of the soot absorption function with the HAB (i.e. with the particle maturity stage) is in fact well consistent with the observations made in wide varied combustion media $[\mathbf{1 7 , 2 3 , 2 6 ]}$ noting that the so-inferred $E(m)$ values are moreover in very good agreement with those estimated in $[\mathbf{2 1 - 2 3 , 2 6 ]}$. Besides, one has to note that in order to get the best fit possible between experimental and modeled data, $\sigma_{\lambda n s}$ has also been adjusted to $1.3 \cdot 10^{-10}, 1.6 \cdot 10^{-10}$ and $1.9 \cdot 10^{-10}$ $\mathrm{cm}^{2 \mathrm{n}-1} \cdot \mathrm{J}^{1-\mathrm{n}}$ at 7, 8 and $9 \mathrm{~mm} \mathrm{HAB}$, respectively, while being kept constant and equal to $4.2 \cdot 10^{-10} \mathrm{~cm}^{2 \mathrm{n}-1} \cdot \mathrm{J}^{1-\mathrm{n}}$ for HAB comprised between 12 and $15 \mathrm{~mm}$. Such an evolution of the multiphoton absorption cross-section in the early soot formation stage is likely to be induced by the internal structure changes undergone by the particles as a function of their maturation stage. This observation is all the more strengthened by the fact that a constant $\sigma_{\lambda n s}$ has to be fixed at high HAB (where mature soot are then formed) noting that the so-derived $4.2 \cdot 10^{-10} \mathrm{~cm}^{2 \mathrm{n}-1} \cdot \mathrm{J}^{1-\mathrm{n}}$ value is furthermore identical to the one assessed in $[\mathbf{2 1}, \mathbf{2 2}]$ based on the analysis of Diesel particles that rapidly reach a mature-like structure.

Concerning the modeling of the soot temperature profiles as a function of the fluence, obtained results are plotted in Fig. 2 for a $\mathrm{HAB}$ of $11 \mathrm{~mm}$ together with measured and simulated data from [23]. As one can see, the model proposed herein satisfactorily reproduces the fluence dependence of the soot temperature below 100 and above $250 \mathrm{~mJ} \cdot \mathrm{cm}^{-2}$ especially considering the uncertainty associated to the experimental data from [23].

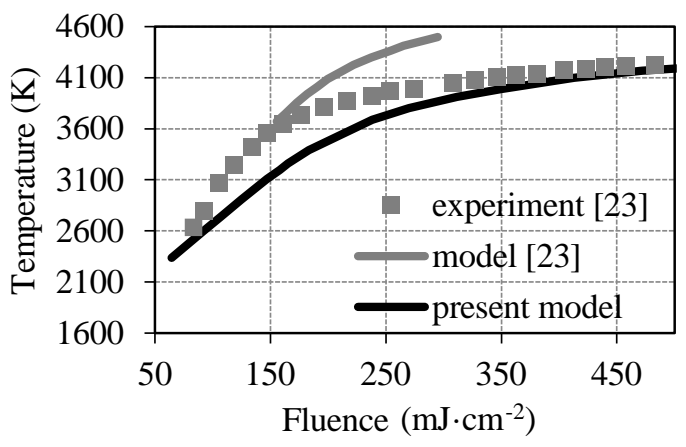

Fig. 2: Comparison between measured and simulated average soot temperature over $10 \mathrm{~ns}$ after the peak of the laser pulse as a function of the laser fluence for a $\mathrm{HAB}$ of $11 \mathrm{~mm}$.

It, however, predicts temperatures lower than those experimentally monitored at intermediate fluences. On the other hand, the model used in [23] globally reproduces well measured data around $150 \mathrm{~mJ} \cdot \mathrm{cm}^{-2}$ while predicting temperatures much higher than their experimental counterparts at high fluences with values even exceeding the sublimation threshold [19]. 
On the whole, more consistent predictions are therefore obtained when using the model proposed in this work noting that several factors are likely to explain the discrepancies observed at intermediate fluences including the accuracy associated to the value of the flame temperatures assessed by NO-LIF thermometry in addition to the uncertainties encompassing the soot temperature assessment process as discussed in [23].

Eventually, modeled LII time decays have been plotted and compared with those reported in [23] after renormalization for HAB of 9 and $12 \mathrm{~mm}$. While a constant $\alpha_{T}$ of 0.37 was selected in [23], the value of the thermal accommodation coefficient has been adjusted as a function of the HAB herein (values of 0.4 and 0.28 being set at 9 and $12 \mathrm{~mm} \mathrm{HAB}$, respectively) as supported by the observations made by López-Yglesias et al. [17] who reported a $\alpha_{T}$ reduction with increasing particle maturity.
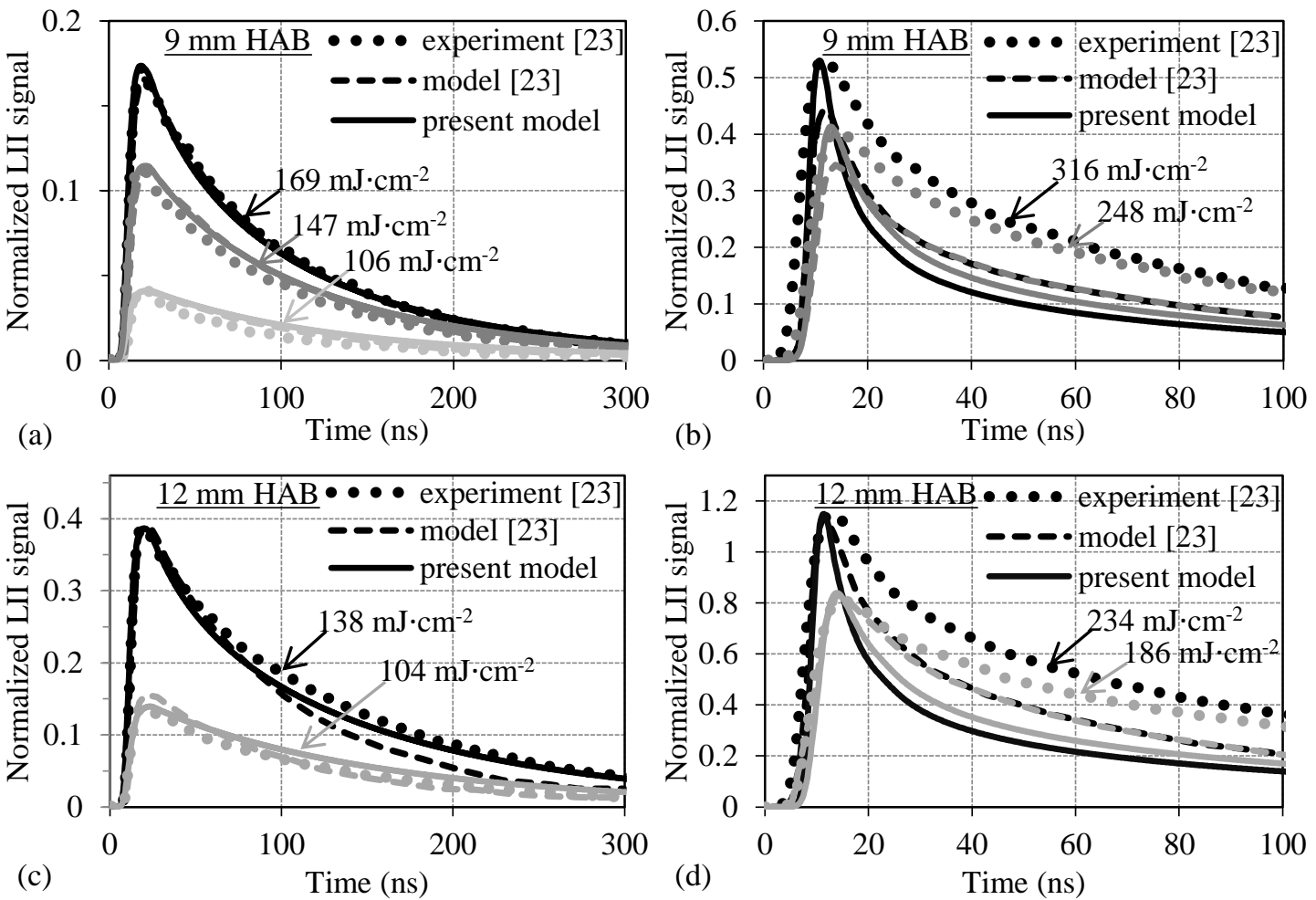

Fig. 3: Measured and modeled LII time decays obtained at various fluences for HAB of 9 (a) and $12 \mathrm{~mm}$ (b).

While both models tend to globally predict well LII time decays measured at $9 \mathrm{~mm} \mathrm{HAB}$ for fluences up to 169 $\mathrm{mJ} \cdot \mathrm{cm}^{-2}$ (Fig. 3(a)), they clearly fail to reproduce experimental data at higher fluences (Fig. 3(b)). At 12 mm HAB (Fig. $3(\mathrm{c})$ and (d)), the model proposed herein allows obtaining a better agreement at low fluence (see the results obtained at $138 \mathrm{~mJ} \cdot \mathrm{cm}^{-2}$ ) even though it still fails to simulate the time-resolved signals recorded at high fluences as is the case of the model used in [23]. That being said, the simulation tool proposed in the present work always correctly predicts the LII peak contrary to the model implemented in [23] thus illustrating a better overall predictive capability. While analyzing the possible factors contributing to the discrepancies observed in the high-fluence regime, it appeared that integrating an additional flux accounting for soot annealing as done in $[\mathbf{2 1 , 2 2 ]}$ could lead to increase the rate of energy production following equation (1) and thus improve the agreement between simulated and measured LII time decays. Such an observation is particularly exemplified in Fig. 4 where the time-resolved signal recorded at $12 \mathrm{~mm} \mathrm{HAB}$ for a fluence of $138 \mathrm{~mJ} \cdot \mathrm{cm}^{-2}$ is compared with simulated time decays obtained when considering or neglecting the annealing process. As one can see, a better fit is then obtained when taking into account the rate of energy increase induced by the 
reorganization of the internal structure of laser-heated soot thus giving interesting insights for future works to be undertaken with the view to improve the predictive character of the LII model implemented herein.

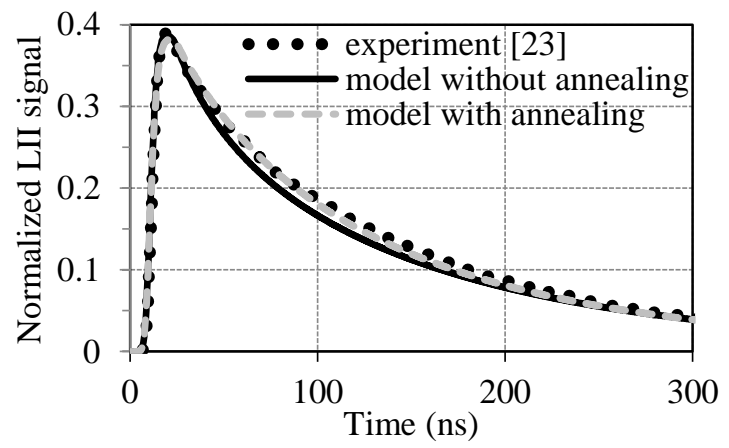

Fig. 4: Comparison between measured and modeled LII time decay at $12 \mathrm{~mm} \mathrm{HAB}$ for a $138-\mathrm{mJ} \cdot \mathrm{cm}^{-2}$ fluence.

\section{Conclusion}

An extensive set of LII signals measured in a $\mathrm{CH}_{4} / \mathrm{O}_{2} / \mathrm{N}_{2}$ premixed flat flame has been simulated within this work using a refined model built upon comprehensive formulations of laser-heated soot heat- and mass-balance equations. While allowing reproducing well LII fluence curves recorded at different HAB in the investigated flame, the model implemented herein also led to theoretical soot temperatures in better agreement with experimentally-monitored ones as compared to the modeling results reported in [23]. Besides, the fitting procedure operated to obtain predicted and measured data merging on a single curve led to infer $E(m)$ and $\sigma_{\lambda n s}$ values increasing with soot maturity. Alternatively, a reduction of the $\alpha_{T}$ value has been assessed with the HAB (i.e. with the soot maturity level). On the whole, LII time decays recorded at low fluences have been well simulated by the model proposed herein as well as by the model used in [23]. These two simulation tools however failed to reproduce data gathered in the high-fluence regime. Additional calculations performed by integrating a complementary rate of energy production through particle annealing eventually allowed improving the agreement between measured and simulated time-resolved LII signals. Such an observation thus traces the route for further developments, currently in progress in our labs, so as to propose more efficient and predictive LII-simulation tools.

\section{Acknowledgements}

The authors warmly thank S. Bejaoui from the NASA - Ames Research Center for providing the fluence curves, not reported in [23], that have been used within the framework of the present modeling study.

\section{References}

[1] P. Desgroux, X. Mercier, K. A. Thomson, "Study of the formation of soot and its precursors in flames using optical diagnostics," Proc. Combust. Inst., vol. 34, pp. 1713-1738, 2013.

[2] H. A. Michelsen, "Probing soot formation, chemical and physical evolution, and oxidation: A review of in situ diagnostic techniques and needs," Proc. Combust. Inst., vol. 36, pp.717-735, 2017.

[3] C. Schulz, B. F. Kock, M. Hofmann, H. Michelsen, S. Will, B. Bougie, R. Suntz, G. Smallwood, "Laser-induced incandescence: recent trends and current questions," Appl. Phys. B, vol. 83, pp. 333-354, 2006.

[4] H. A. Michelsen, C. Schulz, G. J. Smallwood, S. Will, "Laser-induced incandescence: Particulate diagnostics for combustion, atmospheric, and industrial applications," Prog. Energ. Combust. Sci., vol. 51, pp. 2-48, 2015.

[5] L. A. Melton, "Soot diagnostics based on laser heating," Appl. Opt., vol. 23, pp. 2201-2208, 1984.

[6] R. L. Vander Wal, T. M. Ticich, "Cavity ringdown and laser-induced incandescence measurements of soot," Appl. Opt., vol. 38, pp. 1444-1451, 1999.

[7] R. L. Vander Wal, T. M. Ticich, A. Brock Stephens, "Can soot primary particle size be determined using laser-induced incandescence ?," Combust. Flame, vol. 116, pp. 291-296, 1999. 
[8] J. Yon, R. Lemaire, E. Therssen, P. Desgroux, A. Coppalle, K. F. Ren, "Examination of wavelength dependent soot optical properties of diesel and diesel/rapeseed methyl ester mixture by extinction spectra analysis and LII measurements," Appl. Phys. B, vol. 104, pp. 253-271, 2011.

[9] H. A. Michelsen, F. Liu, B. F. Kock, H. Bladh, A. Boiarciuc, M. Charwath, T. Dreier, R. Hadef, M. Hofmann, J. Reimann, S. Will, P. -E. Bengtsson, H. Bockhorn, F. Foucher, K. -P. Geigle, C. Mounaïm-Rousselle, C. Schulz, R. Stirn, B. Tribalet, R. Suntz, "Modeling laser-induced incandescence of soot: a summary and comparison of LII models," Appl. Phys. B, vol. 87, pp. 503-521, 2007.

[10] R.W. Weeks, W. W. Duley, “Aerosol-particle size from light emission during excitation by $\mathrm{TEA} \mathrm{CO}_{2}$ laser pulses," $J$. Appl. Phys., vol. 45, pp. 4661-4662, 1974.

[11] A. C. Eckbreth, "Effects of laser-modulated particulate incandescence on Raman scattering diagnostics," J. Appl. Phys., vol. 48, pp. 4473-4479, 1977.

[12] V. S. Burakov, N. G. Kondrashov, A. A. Stavrov, I. S. Zakharova, "Laser heating and sublimation of carbon particles in a flame," J. Eng. Phys., vol. 32, pp. 584-591, 1977.

[13] G. J. Smallwood, D. R. Snelling, F. Liu, Ö. L. Gülder, "Clouds over soot evaporation: Errors in modeling laser-induced incandescence of soot," J. Heat Transfer, vol. 123, pp. 814-818, 2001.

[14] H. A. Michelsen, "Understanding and predicting the temporal response of laser-induced incandescence from carbonaceous particles," J. Chemical Phys., vol. 118, pp. 7012-7045, 2003.

[15] F. Liu, K. J. Daun, D. R. Snelling, G. J. Smallwood, "Heat conduction from a spherical nano-particle: status of modeling heat conduction in laser-induced incandescence," Appl. Phys. B, vol. 83, pp. 355-382, 2006.

[16] F. Liu, M. Yang, F. A. Hill, D. R. Snelling, G. J. Smallwood, "Influence of polydisperse distributions of both primary particle and aggregate size on temperature in low-fluence LII," Appl. Phys. B, vol. 83, pp. 383-395, 2006.

[17] X. López-Yglesias, P.E. Schrader, H.A. Michelsen, "Soot maturity and absorption cross sections," J. Aerosol Sci., vol. 75, pp. 43-64, 2014.

[18] R. Lemaire, M. Mobtil, "Modeling laser-induced incandescence of soot: a new approach based on the use of inverse techniques," Appl. Phys. B, vol. 119, pp. 577-606, 2015.

[19] F. Goulay, P. E. Schrader, X. López-Yglesias, H. A. Michelsen, "A data set for validation of models of laser-induced incandescence from soot: temporal profiles of LII signal and particle temperature," Appl. Phys. B, vol. 112, pp. 287-306, 2013.

[20] R. Mansmann, T. Terheiden, P. Schmidt, J. Menser, T. Dreier, T. Endres, C. Schulz, "LIISim: a modular signal processing toolbox for laser-induced incandescence measurements," Appl. Phys. B, vol. 124, pp. 69 1-20, 2018.

[21] S. Menanteau, R. Lemaire, "Influence of the expression used to account for the heat conduction flux when modeling laser-induced incandescence of soot aggregates produced in a turbulent flame of Diesel," in Proceedings of the XII International Conference on Computational Heat, Mass and Momentum Transfer 2019, Rome, Italy, 2019.

[22] S. Menanteau, R. Lemaire, "Analysis of the influence of the conduction sub-model formulation on the modeling of laser-induced incandescence of Diesel soot aggregates," Entropy, vol. 22, 21, 2020.

[23] S. Bejaoui, S. Batut, E. Therssen, N. Lamoureux, P. Desgroux, F. Liu, "Measurements and modeling of laser-induced incandescence of soot at different heights in a flat premixed flame," Appl. Phys. B, vol. 118, pp. 449-469, 2015.

[24] H. A. Michelsen, M.A. Linne, B.F. Kock, M. Hofmann, B. Tribalet, C. Schulz, "Modeling laser-induced incandescence of soot: enthalpy changes during sublimation, conduction, and oxidation," Appl. Phys. B, vol 93, pp. 645-656, 2008.

[25] N. A. Fuchs, "On the stationary charge distribution on aerosol particles in a bipolar ionic atmosphere," Geophys. Pura Appl., vol. 56., pp. 185-193, 1963.

[26] H. Bladh, J. Johnsson, N.-E. Olofsson, A. Bohlin, P.-E. Bengtsson, "Optical soot characterization using two-color laserinduced incandescence (2C-LII) in the soot growth region of a premixed flat flame," Proc. Combust. Inst., vol. 33, pp. 641-648, 2011. 